Erratum

\title{
Development of SCAR markers for germplasm characterisation in olive tree (Olea europea L.)
}

Matteo Busconi ${ }^{1 * *}$, Luca Sebastiani ${ }^{2}$ and Corrado Fogher ${ }^{1}$

${ }^{1}$ Istituto di Botanica e Genetica Vegetale, Università Cattolica Sacro Cuore, via Emilia Parmense 84, 29100 Piacenza, Italy; ${ }^{2}$ Scuola Superiore Sant'Anna di Studi Universitari e di Perfezionamento, Piazza Martiri della Libertà, 56127 Pisa, Italy; * Author for correspondence (Phone: + 39-0523-599209; Fax: + 39-0523-599283; e-mail: ist.geneticavegetale@unicatt.it)

Molecular Breeding (2006) 17: 59-68

DOI $10.1007 / \mathrm{s} 11032-005-1395-3$

The authors' affiliations were misrepresented on the title page of the original. 